\title{
FATORES DE RISCO MATERNOS ASSOCIADOS À PREMATURIDADE EM UMA MATERNIDADE-ESCOLA
}

MATERNAL RISK FACTORS ASSOCIATED WITH PREMATURITY IN A UNIVERSITY MATERNITY HOSPITAL

FACTORES DE RIESGO MATERNOS ASOCIADOS CON LA PREMATURIDAD EN UNA MATERNIDAD ESCUELA

\author{
Sthefani da Costa Penha \\ Nathália Patrício Rebouças 2 \\ Ana Vaneska Passos Meireles ${ }^{3}$ \\ Antônio Augusto Ferreira Carioca ${ }^{4}$ \\ Maria Soraia Pinto 5 \\ Natália Sales de Carvalho 6
}

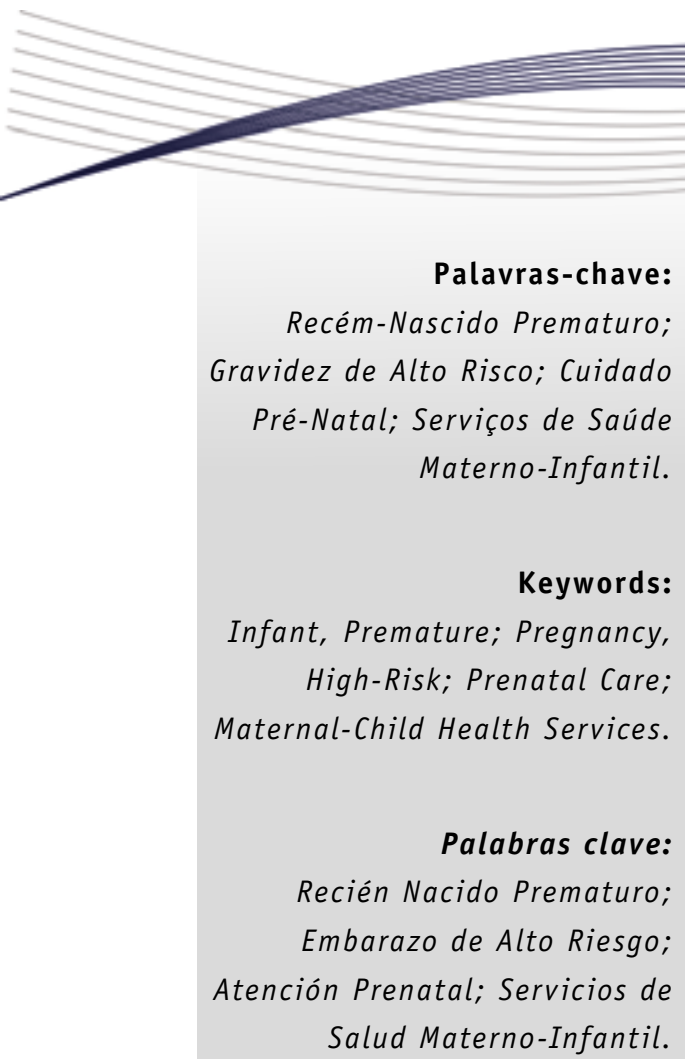

Submetido: $17 / 04 / 2019$

Aprovado: $19 / 10 / 2019$

Autor(a) para Correspondência: Sthefani da Costa Penha R. Dr. Freire Alemão, 225 Mont Serrat - Porto Alegre (RS) 90450-060

E-mail: sthefani.costa@ hotmail.com

\section{RESUMO}

Este estudo teve por objetivo identificar os fatores de risco maternos relacionados à prematuridade em uma maternidade-escola de Fortaleza (CE). Trata-se de pesquisa observacional, transversal e quantitativa, realizada por meio da análise dos prontuários de puérperas e recém-nascidos no periodo de fevereiro a julho de 2018. Tal análise incluiu 100 neonatos pré-termo e 100 a termo e suas respectivas mães. Foram coletados dados de identificação, sociodemográficos, obstétricos, antropométricos e clínicos por meio de um formulário. Eles foram analisados no software SPSS, versão 20.0, com teste de qui-quadrado (significância $p<0,05$ ). Constatou-se associação entre gestações duplas e prematuridade $(p<0,001)$; menor exposição a pré-eclâmpsia e eclâmpsia e recém-nascidos a termo ( $p=$ $0,026) ; e \geq 6$ ou mais consultas pré-natal e partos a termo $(p<0,001)$. Gestações duplas, pré-eclâmpsia e cuidado pré-natal inadequado foram identificados como fatores maternos de risco de prematuridade e, diante disso, a investigação precoce de possiveis condições de risco mediante o cuidado pré-natal se mostra imprescindivel para a prevenção, a redução e/ ou o tratamento de complicações de alto risco relacionadas ao nascimento pré-termo.

\footnotetext{
1. Nutricionista. Aluna de Especialização em Nutrição Materno-Infantil no Instituto de Pesquisas, Ensino e Gestão em Saúde (iPGS).E-mail: sthefani.costa@hotmail.com ORCID: https://orcid.org/0000-0002-1183-4997 2. Nutricionista. Aluna de Especialização em Nutrição Materno-Infantil no iPGS. E-mail: nathaliaprnutri@ outlook.com ORCID: https://orcid.org/0000-0002-3004-0439

3. Nutricionista. Mestre em Ciências Fisiológicas pela Universidade Estadual do Ceará (UECE). Professora na Universidade de Fortaleza (Unifor). E-mail: anavaneskapm@yahoo.com.br 0RCID: https://orcid.org/0000-00030927-5281

4. Nutricionista. Doutor em Nutrição em Saúde Pública pela Universidade de São Paulo (USP). Professor na Unifor. E-mail: nutriaugusto@gmail.com 0RCID: https://orcid.org/0000-0002-1194-562X

5. Nutricionista. Aluna de Doutorado em Saúde Pública na Universidade Federal do Ceará (UFC). Professora na Unifor. E-mail: soraia@unifor.br ORCID: https://orcid.org/0000-0002-9419-746X

6. Nutricionista. Mestre em Nutrição e Saúde pela UECE. Professora na Unifor. E-mail: natycarvalho_@hotmail. com ORCID: https://orcid.org/0000-0003-1069-8644
} 


\section{ABSTRACT}

This study aimed to identify maternal risk factors related to prematurity in a university maternity hospital in Fortaleza, Ceará, Brazil. This is an observational, cross-sectional, and quantitative research, conducted by analyzing the medical records of puerperal women and newborn infants within the period from February to July 2018. Such analysis included 100 preterm and 100 full-term infants and their respective mothers. Identification, sociodemographic, obstetric, anthropometric, and clinical data were collected by means of a form. They were analyzed using the software SPSS, version 20.0, with chi-square test (significance $p<0.05$ ). There was association between double pregnancies and prematurity $(p<0.001)$; lower exposure to preeclampsia and eclampsia and full-term newborn infants $(p=0.026)$; and $\geq 6$ prenatal check-ups and full-term deliveries $(p<0.001)$. Double pregnancies, preeclampsia, and inadequate prenatal care were identified as maternal risk factors for prematurity, therefore, early investigation of potential risk conditions through prenatal care shows to be a must for prevention, reduction, and/or treatment of high-risk complications related to preterm birth.

\section{RESUMEN}

Este estudio tuvo como objetivo identificar los factores de riesgo maternos relacionados con la prematuridad en una maternidad escuela en Fortaleza, Ceará, Brasil. Esta es una investigación observacional, transversal y cuantitativa, realizada mediante el análisis de los registros médicos de las puérperas y los recién nacidos dentro del período de febrero a julio de 2018. Dicho análisis incluyó a 100 recién nacidos prematuros y 100 a término y sus respectivas madres. Los datos de identificación, sociodemográficos, obstétricos, antropométricos y clínicos se recopilaron mediante un formulario. Se analizaron utilizando el software SPSS, versión 20.0, con prueba de chi cuadrado (significancia $p<0,05)$. Hubo asociación entre embarazos dobles y prematuridad $(p<0,001)$; menor exposición a preeclampsia y eclampsia y recién nacidos a término $(p=0,026) ; y \geq 6$ chequeos prenatales y partos a término $(p<0,001)$. Embarazos dobles, preeclampsia y atención prenatal inadecuada se identificaron como factores de riesgo maternos para la prematuridad y, por lo tanto, la investigación temprana de posibles condiciones de riesgo a través de la atención prenatal demuestra ser imprescindible para la prevención, reducción y/o tratamiento de complicaciones de alto riesgo relacionadas con parto prematuro.

\section{INTRODUÇÃO}

A gravidez de alto risco é caracterizada por intercorrências, correspondendo a $20 \%$ das gestações. Ela pode decorrer de fatores sociodemográficos (sobressaindo-se as extremidades de idade, a baixa escolaridade, a ocupação, o estado civil e o uso de drogas lícitas e/ou ilícitas) e nutricionais (o peso pré-gestacional e o ganho de peso gestacional constituem influências diretas no ciclo gravídico, o que se justifica pelo fornecimento das calorias e dos nutrientes necessários para a formação e a alimentação fetal) ${ }^{1-3}$.

Além disso, condições clínicas preexistentes (como diabetes mellitus [DM], hipertensão arterial sistêmica [HAS] e doenças cardiovasculares) ou adquiridas no decorrer do ciclo gestacional (entre elas as sindromes hipertensivas da gravidez [SHG], como pré-eclâmpsia, eclâmpsia e síndrome de HELLP, diabetes mellitus gestacional [DMG] e infecção do trato urinário [ITU]) interferem nessas circunstâncias ${ }^{1,4}$.

Nesse contexto, o aumento ponderal materno excessivo pode acarretar limitação no desenvolvimento do bebê no interior do útero, parto precoce e elevação dos índices de óbito no período entre a concepção e o pós-nascimento ${ }^{5}$. Já a ocorrência de SHG apresenta maior etiologia para morbimortalidade materna e fetal, como nascimentos pré-termo e a consequente necessidade de cuidados em unidade de terapia intensiva neonatal (UTIN). Além disso, a DMG está relacionada a alterações hipertensivas e prematuridade subsequentes ${ }^{6-7}$.

A prematuridade se refere ao parto ocorrido antes das 37 semanas gestacionais completas. Em escala mundial, cerca de 15 milhões de crianças apresentam nascimento pré-termo e o Brasil se encontra entre os 10 primeiros países com maiores taxas de parto precoce. Segundo dados do Sistema de Informação sobre Nascidos Vivos (SINASC), em 2016, a taxa nacional de partos prematuros foi de $11,11 \%$ (sendo $11,28 \%$ na região Nordeste e $12,38 \%$ no Estado do (eará) $)^{8-9}$.

Diante disso, a identificação precoce de determinadas condições é indispensável para tratá-las ou reduzi-las. Nesse contexto, visando à diminuição das taxas de morbimortalidade materna e infantil, criou-se, no âmbito do Sistema Único de 
Saúde (SUS), a estratégia Rede Cegonha - com o intuito de proporcionar atenção humanizada tanto à mulher (para o planejamento reprodutivo, a gestação e o puerpério) quanto à criança na faixa etária de 0 a 24 meses (para o nascimento seguro e o crescimento e o desenvolvimento saudáveis ${ }^{10}$.

Partindo de tal pressuposto, este estudo teve por objetivo:

- Identificar os fatores de risco maternos relacionados à prematuridade em uma maternidadeescola de Fortaleza (CE).

\section{METODOLOGIA}

Trata-se de pesquisa observacional e transversal, com abordagem quantitativa, realizada a partir da leitura e análise dos registros contidos nos prontuários de puérperas e recém-nascidos que estiveram internados em uma das unidades de neonatologia e/ou naqueles disponíveis no Setor de Arquivo Médico e Estatístico (SAME) de uma maternidade-escola na capital do Ceará, com coleta de dados realizada no período entre e fevereiro e julho de 2018.

A quantificação da amostra total foi obtida por meio da fórmula de população finita:

$$
n=N{ }^{*} Z 2^{*} p^{*} q / d 2 *(N-1)+Z 2{ }^{*} p^{*} q
$$

onde $\mathrm{p}=$ prevalência; $Z$ = valor de $Z$ para $u m$ nível de significância; $d=$ precisão desejada; $q$ = quantidade de erro esperada; e $\mathrm{N}=$ universo amostral. Para tal, adotou-se a prevalência de $8,7 \%$ e $5 \%$ de precisão.

Para o cálculo da prevalência, considerou-se que nasceram 5.187 crianças na maternidade estudada (no ano de 2017). Destas, 1.117 nasceram antes de completar 37 semanas gestacionais e 453 recémnascidos pré-termo foram admitidos em setores neonatais: UTIN, unidade de cuidado intermediário neonatal convencional (UCINCo), unidade de cuidado intermediário neonatal canguru (UCINCa) ou alojamento conjunto $(A C)^{11}$.

Assim, a amostra deste estudo foi composta por 400 indivíduos (200 recém-nascidos e 200 puérperas), divididos em 2 grupos: a) recém-nascidos pré-termo (100 neonatos pré-termo, com menos de 36 semanas e 4 dias gestacionais, e as 100 mães respectivas); e b) recém-nascidos a termo (100 neonatos a termo, com 37 semanas gestacionais ou mais, e as 100 mães respectivas).

\section{...Rede Cegonha - com o intuito de proporcionar atenção humanizada tanto à mulher (...) quanto à criança na faixa etária de 0 a 24 meses...}

0s critérios de inclusão foram: recém-nascidos pré-termo e a termo, internados em uma das unidades neonatais da maternidade, e suas mães. Excluíram-se da pesquisa puérperas e neonatos cujas informações indispensáveis para este estudo estivessem incompletas em seus prontuários.

Os dados das mães se elencaram em variáveis categóricas: 1) dados de identificação (iniciais do nome; número do formulário; número do prontuário); 2) dados sociodemográficos (data de nascimento; ocupação; estado civil; escolaridade; etilismo; tabagismo; uso de drogas ilícitas; antecedentes clínicos; uso de medicamentos); e 3) dados obstétricos (gestações anteriores; partos anteriores; abortos anteriores; tipo de parto; tipo de gestação; complicações na gestação; realização de pré-natal; número de consultas pré-natal; idade gestacional em que iniciou o pré-natal). Além disso, adotaramse variáveis contínuas: 4) dados antropométricos (peso pré-gestacional; estatura; Índice de massa corporal [IMC] pré-gestacional e classificação ${ }^{12}$; peso na última consulta pré-natal; idade gestacional do último peso; IMC da última consulta pré-natal e classificação ${ }^{13}$; ganho de peso total da gestação e classificação $0^{12}$ ).

Quanto aos dados do recém-nascido, incluíram-se uma variável categórica - 1) dados de identificação (sexo; data de nascimento) - e uma variável contínua - 2) dados antropométricos (idade gestacional ao nascer e classificação $\left.0^{14}\right)$. Assim, na coleta de dados, empregou-se um formulário estruturado com questões de múltipla escolha.

0 procedimento de tabulação de dados foi conduzido no software Microsoft Office Excel, versão 2013. A análise de dados foi conduzida no software SPSS, versão 20.0. Para as variáveis categóricas, a análise descritiva incluiu a distribuição de frequência relativa; para as variáveis numéricas, foram calculados os valores de média. 0 teste empregado para avaliar as relações foi 0 quiquadrado (significância $p<0,05$ ). 
Esta pesquisa seguiu todos os preceitos da Resolução n. 466/2012, do Conselho Nacional de Saúde (CNS), e foi aprovada pelo Comitê de Ética em Pesquisa (CEP) da Maternidade-Escola Assis Chateaubriand (MEAC), sob Certificado de Apresentação para Apreciação Ética (CAAE) n. 76737317.0.0000.5050 e o Parecer n. 2.556.948/2018. Visto que o estudo foi realizado por meio da leitura e análise de prontuários, sem necessidade de contato direto com os participantes, recorreu-se ao Termo de Fiel Depositário, devidamente assinado pelo diretor-geral da instituição hospitalar antes do início da coleta de dados.

\section{RESULTADOS E DISCUSSÃO}

A população estudada apresentou predomínio de mães na faixa etária entre 19 e 35 anos $(n=127-63,5 \%)$, do $\operatorname{lar}(n=104-52 \%)$, em união estável $(n=94-47 \%)$, com escolaridade até o Ensino Médio completo ( $n=$ $107-53,5 \%)$, não etilistas $(n=192-96 \%)$, não tabagistas $(n=193-96,5 \%)$ e não usuárias de drogas ilícitas $(n=191-95,5 \%)$ e não houve diferença estatística entre as variáveis analisadas para ambos os grupos (Tabela 1).

Tabela 1 - Dados sociodemográficos das puérperas associados a nascimentos pré-termo e a termo em uma maternidade. Fortaleza, 2018.

\begin{tabular}{|c|c|c|c|c|}
\hline Variáveis & $\begin{array}{c}\text { Pré-termo } \\
(\%)\end{array}$ & $\begin{array}{c}\text { A termo } \\
(\%)\end{array}$ & $\begin{array}{l}\text { Total } \\
(\%)\end{array}$ & $\mathbf{P} *$ \\
\hline Idade & & & & 0,471 \\
\hline$<19$ anos & 11 & 17 & 14 & \\
\hline Entre 19 e 35 anos & 66 & 61 & 63,5 & \\
\hline$>35$ anos & 23 & 22 & 22,5 & \\
\hline 0 cupação & & & & 0,433 \\
\hline Emprego fixo & 38 & 35 & 36,5 & \\
\hline Do lar & 52 & 52 & 52 & \\
\hline Estudante & 10 & 13 & 11,5 & \\
\hline Estado civil & & & & 0,353 \\
\hline Casada & 29 & 23 & 26 & \\
\hline União estável & 48 & 46 & 47 & \\
\hline Solteira & 23 & 31 & 27 & \\
\hline Escolaridade & & & & 0,906 \\
\hline Ensino Fundamental & 41 & 38 & 39,5 & \\
\hline Ensino Médio & 52 & 55 & 53,5 & \\
\hline Ensino Superior & 7 & 7 & 7 & \\
\hline Etilismo & & & & 0,470 \\
\hline Sim & 3 & 5 & 4 & \\
\hline Não & 97 & 95 & 96 & \\
\hline Tabagismo & & & & 0,700 \\
\hline $\operatorname{Sim}$ & 4 & 3 & 3,5 & \\
\hline Não & 96 & 97 & 96,5 & \\
\hline Uso de drogas ilicitas & & & & 0,733 \\
\hline Sim & 4 & 5 & 4,5 & \\
\hline Não & 96 & 95 & 95,5 & \\
\hline
\end{tabular}

* Teste de qui-quadrado.

No entanto, nas faixas etárias de risco, prevaleceram mães de recém-nascidos pré-termo com idade acima de $35 \operatorname{anos}(n=23-23 \%)$. Tal achado demonstra que a proporção estudada de nascimentos prematuros apresenta diferenças nas faixas etárias maiores, corroborando estudo realizado em 2007-2008, com gestantes atendidas 
nos estabelecimentos do SUS do Município do Rio de Janeiro, que indica maior risco de parto pré-termo à medida que a idade da mulher avança ${ }^{15}$.

Ademais, averiguou-se maior proporção de puérperas do $\operatorname{lar}(n=52-52 \%$ ), em união estável ( $n=48$ - 48\%) e com Ensino Médio ( $n=52$ - 52\%), concordando com outros autores, os quais apresentaram como população prevalente mulheres sem atividade remunerada e com companheiro fixo, diferenciando-se quanto à escolaridade (classificada como baixa) ${ }^{16}$. E, ainda, identificaram-se mães não etilistas, não tabagistas e não usuárias de drogas ilícitas, o que se contrapõe aos achados de outra pesquisa, cujo objetivo foi investigar o perfil das condições de saúde de mães com partos prematuros, na qual se evidenciou o uso de tais substâncias e partos pré-termo ${ }^{17}$.

Ao analisar os antecedentes clínicos, houve prevalência de mães de recém-nascidos prematuros com DM $(n=17-17 \%)$ e HAS $(n=16-16 \%)$ (Tabela 2$)$, confirmando resultados de estudo transversal e descritivo, o qual caracterizou o perfil de recém-nascidos atendidos na UTIN de um hospital estadual, identificando neonatos pré-termo com mães portadoras de DM e HAS ${ }^{18}$. 0 desfecho pode ser justificado pela maior exposição a resistência insulínica, aumento dos níveis pressóricos e estresse metabólico.

Tabela 2 - Antecedentes clínicos e história reprodutiva de puérperas associados a nascimentos pré-termo e a termo em uma maternidade. Fortaleza, 2018.

\begin{tabular}{|c|c|c|c|c|}
\hline Variáveis & $\begin{array}{c}\text { Pré-termo } \\
(\%)\end{array}$ & $\begin{array}{c}\text { A termo } \\
(\%)\end{array}$ & $\begin{array}{c}\text { Total } \\
(\%)\end{array}$ & $P *$ \\
\hline \multicolumn{5}{|l|}{ Antecedentes clínicos } \\
\hline DM & 17 & 8 & 12,5 & 0,054 \\
\hline HAS & 16 & 13 & 14,5 & 0,547 \\
\hline Hipotireoidismo & 5 & 1 & 3 & 0,097 \\
\hline Anemia & 3 & 2 & 2,5 & 0,651 \\
\hline Sifilis & 2 & 2 & 2 & 0,100 \\
\hline Cardiopatia & 1,01 & 1 & 1,01 & 0,994 \\
\hline Uso de medicamentos & 70 & 62 & 66 & 0,232 \\
\hline Gestações anteriores & & & & 0,808 \\
\hline Nenhuma & 34 & 38 & 36 & \\
\hline $1-3$ & 53 & 51 & 52 & \\
\hline$>3$ & 13 & 11 & 12 & \\
\hline Partos anteriores & & & & 0,827 \\
\hline Nenhum & 42 & 46 & 44 & \\
\hline $1-3$ & 53 & 50 & 51,5 & \\
\hline$>3$ & 5 & 4 & 4,5 & \\
\hline Abortos anteriores & & & & 0,556 \\
\hline Nenhum & 71,71 & 74 & 72,86 & \\
\hline $1-3$ & 27,27 & 26 & 26,63 & \\
\hline$>3$ & 1,01 & 0 & 0,5 & \\
\hline
\end{tabular}

* Teste de qui-quadrado; DM = diabetes mellitus; HAS = hipertensão arterial sistêmica.

Quanto à história reprodutiva, as mães apresentaram de 1 a 3 gestações anteriores $(n=53-53 \%)$, de 1 a 3 partos anteriores $(n=53-53 \%$ ) e nenhum aborto anterior ( $n=71-71,71 \%$ ), não havendo diferença estatística na amostra (Tabela 2), o que se assemelha ao encontrado em uma pesquisa realizada entre janeiro e junho de 2015, cujo objetivo foi identificar o perfil epidemiológico de gestantes de alto risco atendidas no Instituto da Mulher do Município de Francisco Beltrão4.

Em relação à gestação atual, houve prevalência de gestação tipo única ( $n=187-93,5 \%$ ) e parto cesáreo ( $n$ $=115-57,5 \%$ ) para os 2 grupos. Contudo, gestações duplas apresentaram a maior associação com a ocorrência de prematuridade $(n=13-13 \%)(p<0,001)$ quando comparadas aos nascimentos a termo (Tabela 3 ), assim como estabelecido na literatura, a qual afirma que gestações múltiplas aumentam - em 17 vezes - as chances 
de ocorrência de nascimentos antes das 37 semanas gestacionais completas, devido à maior exposição a intercorrências durante o período gestacional ${ }^{19}$.

Tabela 3 - Dados obstétricos e complicações gestacionais associados a nascimentos pré-termo e a termo em uma maternidade. Fortaleza, 2018.

\begin{tabular}{|c|c|c|c|c|}
\hline Variáveis & $\begin{array}{c}\text { Pré-termo } \\
(\%)\end{array}$ & $\begin{array}{c}\text { A termo } \\
(\%)\end{array}$ & $\begin{array}{c}\text { Total } \\
(\%) \\
\end{array}$ & $\mathbf{P}$ * \\
\hline Tipo de gestação & & & & $<0,001$ \\
\hline Única & 87 & 100 & 93,5 & \\
\hline Dupla & 13 & 0 & 6,5 & \\
\hline Tipo de parto & & & & 0,198 \\
\hline Normal & 38 & 47 & 42,5 & \\
\hline Cesário & 62 & 53 & 57,5 & \\
\hline Realização de pré-natal & & & & 0,312 \\
\hline Sim & 97 & 99 & 98 & \\
\hline Não & 3 & 1 & 2 & \\
\hline Número de consultas pré-natal & & & & $<0,001$ \\
\hline Nenhuma & 3 & 0 & 1,5 & \\
\hline $1-3$ & 29 & 7 & 18 & \\
\hline $4-6$ & 38 & 28 & 33 & \\
\hline$>6$ & 30 & 65 & 47,5 & \\
\hline Trimestre de início pré-natal & & & & 0,156 \\
\hline $1^{\circ}$ trimestre & 70,1 & 64 & 67,01 & \\
\hline $2^{\circ}$ trimestre & 28,87 & 30 & 29,44 & \\
\hline $3^{\circ}$ trimestre & 1,03 & 6 & 3,55 & \\
\hline \multicolumn{5}{|l|}{ Complicações gestacionais } \\
\hline ITU & & & & 0,881 \\
\hline Sim & 34 & 33 & 33,5 & \\
\hline Não & 66 & 67 & 66,5 & \\
\hline Pré-eclâmpsia/eclâmpsia & & & & 0,026 \\
\hline Sim & 34 & 20 & 27 & \\
\hline Não & 66 & 80 & 73 & \\
\hline DMG & & & & 0,809 \\
\hline Sim & 9 & 10 & 9,5 & \\
\hline Não & 91 & 90 & 90,5 & \\
\hline Anemia & & & & 0,248 \\
\hline Sim & 5 & 33 & 3,5 & \\
\hline Não & 95 & 67 & 96,5 & \\
\hline Síndrome de HELLP & & & & 0,155 \\
\hline Sim & 2 & 0 & 1 & \\
\hline Não & 98 & 100 & 99 & \\
\hline Sífilis & & & & 0,316 \\
\hline Sim & 1 & 0 & 0,5 & \\
\hline Não & 99 & 100 & 99,5 & \\
\hline
\end{tabular}

* Teste de qui-quadrado; ITU = infecção do trato urinário; DMG = diabetes mellitus gestacional.

0 parto cesáreo, também, esteve mais presente em nascimentos pré-termo ( $n=62-62 \%$ ), concordando com estudos realizados em Teresina (PI), em 2012 e 2013, e em Porto Alegre (RS), em 2012, sugerindo que intercorrências gestacionais levam à gravidez de alto risco, sendo, na maioria das vezes, precursoras de partos prematuros e da consequente indicação de cesárea ${ }^{8,16}$.

As principais complicações gestacionais observadas foram ITU ( $n=67-33,5 \%)$ e pré-eclâmpsia e eclâmpsia 
( $n=54-27 \%$ ) para ambos os grupos. No entanto, averiguou-se que menor exposição de mães a pré-eclâmpsia e eclâmpsia apresentou relação direta com nascimentos de recém-nascidos a termo $(n=80-80 \%)(p=0,026)$, semelhante aos achados de outros autores, que evidenciaram maior frequência de pré-eclâmpsia entre as intercorrências maternas correlacionadas à prematuridade ${ }^{16}$. Isso pode ser explicado pela indicação de ITU não tratada adequadamente no pré-natal como fator predisponente ao surgimento de pielonefrite (infecção nos rins), causando dano tecidual por endotoxinas bacterianas e disfunção da pressão sanguínea, propiciando, assim, maior risco de surgimento de pré-eclâmpsia ${ }^{18,20}$.

Quanto ao pré-natal, $98 \%(n=196)$ das mulheres foram acompanhadas, predominando um número de consultas $\geq 6(n=95-47,5 \%)$ e a maioria das que receberam atenção iniciaram no primeiro trimestre gestacional ( $n=132$ - 67,01\%). Além disso, observou-se que $65 \%(n=65)$ das puérperas realizaram $\geq 6$ consultas pré-natal e eram mães de recém-nascidos a termo $(p<0,001)$, apresentando correlação significativa entre atenção pré-natal adequada e prevenção de nascimentos prematuros (Tabela 3), confirmando resultados de pesquisa desenvolvida no município de Maringá (PR), em 2009, os quais indicam que a atenção pré-natal adequada, por meio de 7 consultas, relaciona-se com menor probabilidade de prematuridade ${ }^{19}$.

Diante disso, a atenção pré-natal adequada é imprescindível para a prevenção de partos prematuros por possibilitar a identificação precoce das condições de risco. A Rede Cegonha se instituiu nesse contexto e tem por objetivo garantir o cuidado humanizado ao público materno-infantil, iniciado desde o planejamento reprodutivo e o atendimento pré-natal, estendendo-se ao parto e ao nascimento seguro, crescimento e desenvolvimento saudáveis da criança, até que ela alcance os 2 anos de vida ${ }^{10,21}$.

No tocante ao estado nutricional, a maioria das gestantes estava com excesso de peso antes da gestação $(n=110-55 \%)$, com a média de IMC $=26,09 \mathrm{~kg} / \mathrm{m}^{2}$, e na última consulta pré-natal $(\mathrm{n}=117-58,5 \%)$, com a média de $I M C=29,27 \mathrm{~kg} / \mathrm{m}^{2}$. Apesar de homogeneidade na amostra, observou-se que mães de recém-nascidos pré-termo apresentaram, no período pré-gestacional, excesso de peso ( $n=54-54 \%)$ e, em relação ao estado nutricional na última consulta pré-natal, averiguou-se que mães de recém-nascidos pré-termo também se encontravam com excesso de peso ( $n=55-55 \%$ ) (Tabela 4$)$.

Tabela 4 - Estado nutricional e ganho de peso total da gestação associados a nascimentos pré-termo e a termo em uma maternidade. Fortaleza, 2018.

\begin{tabular}{|c|c|c|c|c|}
\hline Variáveis & $\begin{array}{c}\text { Pré-termo } \\
(\%)\end{array}$ & $\begin{array}{c}\text { A termo } \\
(\%)\end{array}$ & $\begin{array}{c}\text { Total } \\
(\%)\end{array}$ & $\mathbf{P} *$ \\
\hline Estado nutricional pré-gestacional & & & & 0,604 \\
\hline Déficit de peso & 4 & 8 & 6 & \\
\hline Peso adequado & 42 & 36 & 39 & \\
\hline Excesso de peso & 54 & 56 & 55 & \\
\hline Estado nutricional do último pré-natal & & & & 0,797 \\
\hline Déficit de peso & 15 & 13 & 14 & \\
\hline Peso adequado & 30 & 25 & 27,5 & \\
\hline Excesso de peso & 55 & 62 & 58,5 & \\
\hline Ganho de peso total da gestação & & & & 0,133 \\
\hline Abaixo do recomendado & 63 & 49 & 56 & \\
\hline Dentro do recomendado & 22 & 29 & 25,5 & \\
\hline Acima do recomendado & 15 & 22 & 18,5 & \\
\hline
\end{tabular}

Tal achado se encontra em concordância com os resultados de estudo realizado no período de abril a setembro de 2015, com mães de recém-nascidos pré-termo em uma maternidade pública em Parnamirim (RN), no qual também se observou semelhança entre maior prevalência de sobrepeso e obesidade antes e durante a gestação e partos pré-termo, determinando que a variável do estado nutricional materno pode ser considerada fator de risco de nascimento prematuro, justificando-se pela modificação do estado metabólico, com proliferação de citocinas inflamatórias e alteração na resistência insulínica ${ }^{17}$. 
No entanto, a prevalência de ganho de peso total da gestação se deu abaixo do recomendado para os 2 grupos $(n=112-56 \%$ ), considerando que as gestantes já iniciaram o pré-natal com a classificação de excesso de peso e a incompletude do período gestacional. Além disso, constatou-se que as mães que expuseram ganho de peso abaixo do recomendado apresentaram maior associação com partos pré-termo ( $n=63-63 \%$ ) (Tabela 4$)$, corroborando uma pesquisa de caso-controle realizada em Campina Grande ( $P B)$, no período de junho de 2008 a maio de 2009, a qual identificou que o ganho ponderal materno insuficiente demonstrou risco de prematuridade ${ }^{22}$.

\section{CONCLUSÃO}

Este estudo evidenciou gestações duplas, pré-eclâmpsia, cuidado pré-natal inadequado e excesso de peso como fatores maternos de risco de prematuridade, podendo resultar em maior prevalência de recém-nascidos prematuros presentes em UTIN e maior tempo de internação, propiciando ao aumento de custos hospitalares.

Desse modo, mostram-se imprescindiveis a melhoria do planejamento de ações profiláticas e a ampliação da cobertura pré-natal, considerando que tal assistência enfatiza cuidados humanizados durante todo o período gestacional, prevenindo, reduzindo e/ou tratando complicações de alto risco que podem estar relacionadas a nascimento prétermo e, assim, colaborar para o aumento da eficácia das estratégias propostas pelo Ministério da Saúde (MS) - como a Rede Cegonha.

\section{CONTRIBUIÇÃO DOS AUTORES}

\section{Sthefani da Costa Penha e Nathália Patricio} Rebouças contribuíram com a realização da pesquisa, o delineamento do estudo e a redação do manuscrito. Ana Vaneska Passos Meireles contribuiu com a redação e a revisão crítica do manuscrito. Antônio Augusto Ferreira Carioca, Maria Soraia Pinto e Natália Sales de Carvalho contribuíram com a revisão crítica do manuscrito.

\section{REFERÊNCIAS}

1. Rodrigues ARM, Dantas SLC, Pereira AMM, Silveira MAM, Rodrigues DP. Gravidez de alto risco: análise dos determinantes de saúde. Sanare (Sobral, Online) [serial on the internet]. 2017 [cited 2018
Feb 28];16(1):23-8. Available from: https://sanare. emnuvens.com.br/sanare/article/view/1135

2. Teixeira MPC, Queiroga TPR, Mesquita MA. Frequência e fatores de risco para o nascimento de recém-nascidos pequenos para idade gestacional em maternidade pública. Einstein (São Paulo) [serial on the internet]. 2016 [cited 2019 Nov 25];14(3):31723. Available from: http://www.scielo.br/pdf/eins/ v14n3/pt 1679-4508-eins-14-03-0317.pdf

3. Ribeiro AM, Lima MC, Lira PIC, Silva GAP. Baixo peso ao nascer e obesidade: associação causal ou casual? Rev Paul Pediatr [serial on the internet]. 2015 [cited 2019 Nov 25];33(3):341-8. Available from: http://www.scielo.br/pdf/rpp/v33n3/01030582-rpp-33-03-0340.pdf

4. Costa LD, Cura CC, Perondi AR, França VF, Bortoloti DS. Perfil epidemiológico de gestantes de alto risco. Cogitare Enferm [serial on the internet]. 2016 [cited 2018 Feb 28];21(2):1-8. Available from: https:// revistas.ufpr.br/cogitare/article/view/44192/28238

5. Teixeira CSS, Cabral ACV. Avaliação nutricional de gestantes sob acompanhamento em serviços de pré-natal distintos: a região metropolitana e o ambiente rural. Rev Bras Ginecol Obstet [serial on the internet]. 2016 [cited 2019 Nov 25];38(1): 2734. Available from: http://www.scielo.br/pdf/rbgo/ v38n1/0100-7203-rbgo-38-01-00027.pdf

6. Souza NL, Araújo ACPF, Costa ICC. Representações sociais de puérperas sobre as síndromes hipertensivas da gravidez e nascimento prematuro. Rev Latinoam Enferm [serial on the internet]. 2013 [cited 2018 Mar 4];21(3):1-8. Available from: http://www.revistas. usp.br/rlae/article/view/75979

7. Amaral AR, Silva JC, Ferreira BS, Silva MR, Bertini AMA. Impacto do diabetes gestacional nos desfechos neonatais: uma coorte retrospectiva. Sci Med. $2015 ; 25(1): 1-6$.

8. Oliveira LL, Gonçalves AC, Costa JSD, Bonilha $A L L$. Fatores maternos e neonatais relacionados à prematuridade. Rev Esc Enferm USP [serial on the internet]. 2016 [cited 2019 Nov 25];50(3):382-9. Available from: http://www.scielo.br/pdf/reeusp/ v50n3/pt 0080-6234-reeusp-50-03-0382.pdf

9. Brasil. Sistema de Informação sobre Nascidos Vivos (SINASC) [homepage on the internet]. 2016 [cited 2018 Mar 11]. Available from: http://tabnet.datasus. gov.br/cgi/deftohtm.exe?sinasc $\% 2 F c n v \% 2 F n v u f . d e f$

10. Brasil. Portaria n. 1.459, de 24 de junho de 2011. Institui no âmbito do Sistema Único de Saúde SUS - a Rede Cegonha [document on the internet]. 2011 [cited 2018 May 28]. Available from: http:// bvsms.saude.gov.br/bvs/saudelegis/gm/2011/ prt1459 2406 2011.html 
11. Maternidade-Escola Assis Chateaubriand. Relatório Institucional Assistencial da MaternidadeEscola Assis Chateaubriand (MEAC) [document on the internet]. 2017 [cited 2018 May 29]. Available from: http://ebserh.gov.br/web/meac-ufc/relatorio-degestao1

12. Institute of Medicine. Weight gain during pregnancy: reexamining the guidelines. Washington (DC): National Academic Press; 2009.

13. Atalah S, Castilho C, Castro R, Amparo A. Propuesta de un nuevo estándar de evaluación nutricional en embarazadas. Rev Méd Chile [serial on the internet]. 1997 [cited 2018 Mar 4];125(12):1429-36. Available form: http://bases.bireme.br/cgi-bin/wxislind.exe/ iah/online/?IsisScript=iah/iah. $x i s \& s r c=$ google \&bas $\mathrm{e}=\mathrm{LILACS} \&$ lang $=p \& n e x t A c t i o n=\ln k \&$ exprSearch $=21$ 0390\&indexSearch $=$ ID

14. Brasil. Manual do Método Canguru: seguimento compartilhado entre a atenção hospitalar e a atenção básica. Brasília (DF): Ministério da Saúde; 2015.

15. Vettore MV, Dias M, Vettore MV, Leal MC. Avaliação da qualidade da atenção pré-natal dentre gestantes com e sem história de prematuridade no Sistema Único de Saúde no Rio de Janeiro, Brasil. Rev Bras Saúde Mater Infant [serial on the internet]. 2013 [cited 2018 Aug 22];13(2):89-100. Available from: http://www.scielo.br/scielo.php?script=sci arttext \&pid $=$ S1519-38292013000200002

16. Gonzaga ICA, Santos SLD, Silva ARV, Campelo V. Atenção pré-natal e fatores de risco associados à prematuridade e baixo peso ao nascer em capital do Nordeste brasileiro. Ciênc Saúde Colet [serial on the internet]. 2016 [cited 2019 Nov 25];21(6):196574. Available from: http://www.scielo.br/pdf/csc/ v21n6/1413-8123-csc-21-06-1965.pdf

17. Teixeira GA, Carvalho JBL, Sena AV, Morais PC, Alves TRM. Características maternas de partos prematuros. Enferm Atual [serial on the internet]. 2017 [cited 2018 Aug 10];81:19-23. Available from: http://revistaenfermagematual.com/arquivos/ ED 81 REVISTA 19/02.pdf

18. Oliveira CS, Casagrande GA, Grecco LC, Golin M0. Perfil de recém-nascidos pré-termo internados na unidade de terapia intensiva de hospital de alta complexidade. ABCS Health Sci [serial on the internet]. 2015 [cited 2018 Mar 10];40(1):28-32. Available from: https://www.portalnepas.org.br/ abcshs/article/view/700

19. Melo WA, Carvalho, MDB. Análise multivariada dos fatores de riscos para prematuridade no Sul do Brasil. Revista Eletrônica Gestão \& Saúde [serial on the internet]. 2014 [cited 2018 Aug 10];5(2):398409. Available from: https://periodicos.unb.br/ index.php/rgs/article/view/436/419
20. Duarte G, Marcolin AC, Quintana SM, Cavalli RC. Infecção urinária na gravidez. Rev Bras Ginecol Obstet [serial on the internet]. 2008 [cited 2019 Nov 25];30(2):93-100. Available from: http://www. scielo.br/pdf/rbgo/v30n2/08.pdf

21. Sampaio ARR, Bousquat A, Barros C. Contato pele a pele ao nascer: um desafio para a promoção do aleitamento materno em maternidade pública no Nordeste brasileiro com o título de Hospital Amigo da Criança. Epidemiol Serv Saúde [serial on the internet]. 2016 [cited 2019 Nov 25];25(2):28190. Available from: http://www.scielo.br/pdf/ress/ v25n2/2237-9622-ress-25-02-00281.pdf

22. Assunção PL, Novaes HMD, Alencar GP, Melo ASO, Almeida MF. Fatores associados ao nascimento prétermo em Campina Grande, Paraíba, Brasil: um estudo de caso-controle. Cad Saúde Pública [serial on the internet]. 2012 [cited 2018 Aug 10];28(6):107890. Available from: http://www.scielo.br/pdf/csp/ v28n6/07.pdf
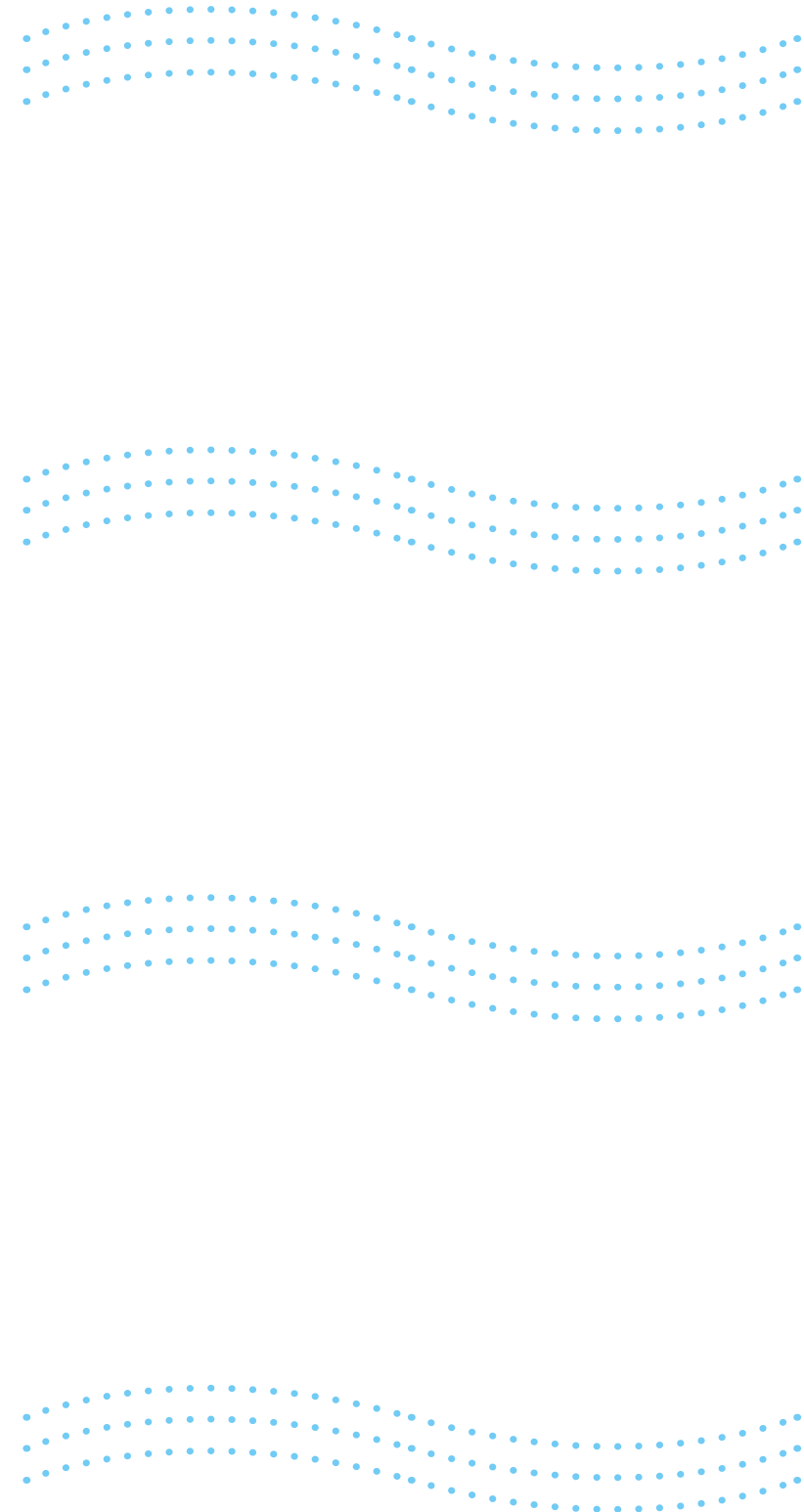

SANARE (Sobral, Online). 2019 Jul-Dec;18(2):43-51 - 51 